\title{
Surface free energy and microarray deposition technology
}

\author{
Glen McHale ${ }^{* a}$ \\ Received 27th November 2006, Accepted 6th February 2007 \\ Postprint version of: Analyst, 2007, 132, 192 - 195 \\ s DOI: 10.1039/b617339j
}

Microarray techniques use a combinatorial approach to assess complex biochemical interactions. The fundamental goal is simultaneous large scale experimentation analogous to the automation achieved in the semiconductor industry. However, microarray deposition inherently involves liquids contacting solid substrates. Liquid droplet shapes are determined by surface and interfacial 10 tension forces, and flows during drying. This article looks at how surface free energy and wetting considerations may influence the accuracy and reliability of spotted microarray experiments.

\section{Introduction}

The microarray concept seeks to provide high-throughput analysis of, e.g., DNA sequences. The concept is not limited 15 to DNA, but can be used for a wide range of other biochemical interactions. Effectively, microarrays are libraries of biological or chemical entities immobilised in a grid/array on a solid surface. The entities are referred to as probes and these probes interact with targets (a gene, mRNA, cDNA, ${ }_{20}$ protein, etc). The extent of interaction is assessed using fluorescent labels, colorimetric/chromogenic labels, radioisotope labels or label-free methods (e.g. scanning Kelvin microscopy, scanning tunnelling microscopy, electrochemical detection). ${ }^{1}$ A common array interrogation 25 method is to use fluorescent confocal microscopy to obtain an integrated intensity signal for each spot on the substrate. Changes in signal intensity across the array provide information about hybridisation. Three common methods of producing microarrays are robotic spotting, lithography and ${ }_{30}$ bead-based assembly. In this article, we focus on the first of these. We explain why we believe there is a need to understand wetting, we identify some of the wetting issues for such microarrays and outline simple principles that can be used to understand accuracy and reproducibility.

\section{The need to understand wetting}

Wetting describes how a liquid interacts with a solid through the change of interfacial areas to minimise the overall surface free energy of a system. To provide an overview of its significance consider the spotted fluorescent microarray in 40 fig. 1. It is evident that spots may show as donuts (hollow ring shapes), that they can be irregular in size, distorted in shape and may have particle/dust contamination and bubbles. All of these are effects due to wetting and surface free energy. Donuts are ring stains produced by the drying of a droplet ${ }_{45}$ having a pinned solid-liquid interface. Irregular shapes and sizes are related to the breaking of liquid bridges between a solid (spotting pin) and a substrate, and to the impact on droplet spreading of the state of the substrate surface (e.g. roughness or chemical heterogeneity).

"School of Biomedical \& Natural Sciences, Nottingham Trent University, Clifion Lane,Nottingham, UK. Fax: +44 (0)115 8486636; Tel: +44 (0)115 8483365; E-mail: glen.mchale@ntu.ac.uk

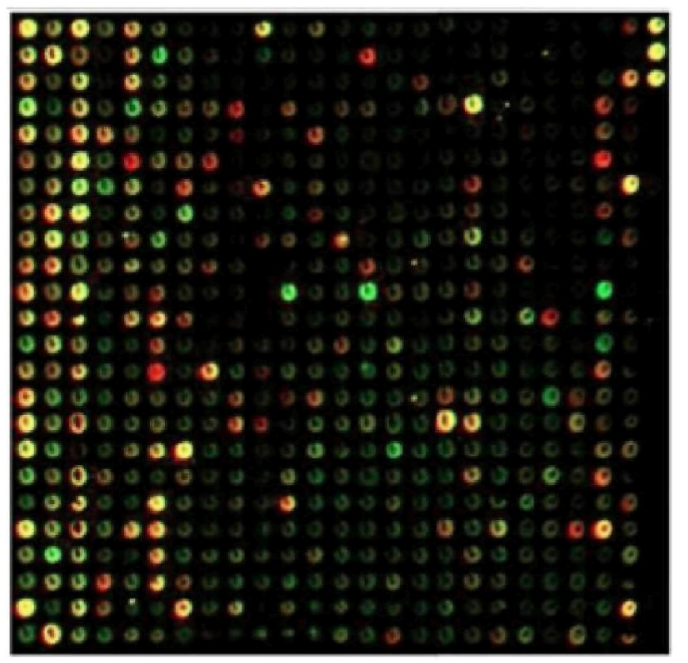

Fig. 1 Spotted microarray showing distortions in spots. Reprinted from ref. [2], Copyright 2003, with permission of Elsevier.

50 A spotted microarray begins with the production of probes (e.g. cDNA via parallel PCR, Oligo probes via oligionucleotide synthesis) and the preparation of a substrate (e.g. a glass slide). ${ }^{3} \mathrm{~A}$ spotting robot creates an array (50-500 $\mu \mathrm{m}$ diameter spots) which is then fixed. The robot dips pins ${ }_{55}$ into wells, deposits droplets onto the substrate and washes the pins. Alternatively, inkjet synthesis can be used with nozzles firing $A, C, G, T$ nucleotides. The array is used with targets created by extracting RNA from tissue, converting the mRNA to cDNA, fluroescently labeled with Cy3 and Cy5. ${ }_{60}$ Hydridisation is followed by a wash step and by image acquisition. This process is shown schematically in fig. 2 .

Spot shape, spot size and concentration are critical parameters in the spotting process. The deposition technologies include pins or needles, pin and ring, and inkjet ${ }_{65}$ printing. Quality issues include print quality, substrate chemistry and hydrophobicity, spotting buffer viscosity, $\mathrm{pH}$, evaporation and probe concentration. Wetting is clearly important in determining the quality of a microarray and yet from around 22000 journal articles with the keyword 70 "microarrays", only 4 are listed when combining this with the keyword "wetting", a further 16 using "contact angle" and none with "surface free energy". 


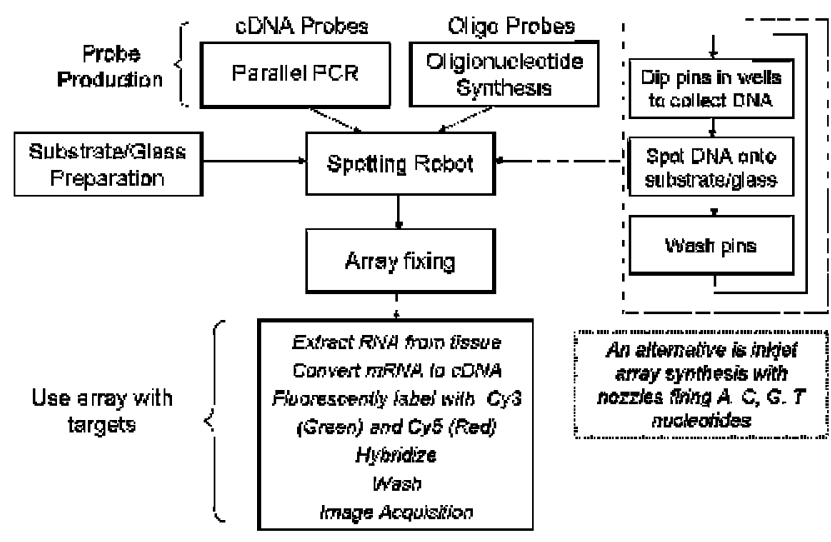

Fig. 2 Spotted DNA microarray formation (after ref [3])

\section{Principles of wetting}

In microarrays, size matters. A liquid surface tries to 75 minimize its surface area. For a free droplet, the minimum surface area shapes it into a sphere, but when a droplet is on a substrate this is no longer the case. Minimizing the surface free energy then involves the solid-liquid and solid-vapor interfacial areas and not just the liquid-vapor interface. Thus, so when a droplet is on, e.g., a wire of a fence, it extends along the wire. Since the force due to surface tension, $\gamma_{L V}$, scales with length and that from gravity scales with length cubed (volume), small droplet size means surface tension wins over gravity and becomes the dominant force. For water, small 85 means less than $10 \%$ of the capillary length $\left(\kappa_{\text {water }}{ }^{-1}=2.73 \mathrm{~mm}\right)$ and so droplets deposited in a microarray are shaped and structured by interfacial free energies.

A droplet deposited onto a flat substrate may spread into a film or stop spreading and remain as a droplet. The surface ${ }_{90}$ free energy per unit area for contact between a smooth and flat solid and a vapor is $\gamma_{S V}$. If we intercalate a thin layer of liquid between the solid and the vapor we now have two interfaces and the energy becomes $\left(\gamma_{S L}+\gamma_{L V}\right)$. A film will form when the difference $S=\left(\gamma_{S L}+\gamma_{L V}\right)-\gamma_{S V}$ (the spreading power) is ${ }_{95}$ positive, unless the liquid is on a curved surface (e.g. capillaries, fibers) or a patterned surface (chemically or topographically). In theory, if $S<0$, a small droplet stops spreading and remains as a spherical cap shape with a contact angle, $\theta_{e}$, to the solid described by Young's law: $100 \cos \theta_{e}=\left(\gamma_{S V}+\gamma_{S L}\right) / \gamma_{L V}$, which summarizes the chemistry. In practice, contact angle hysteresis means no motion of the contact perimeter occurs until some advancing contact angle is exceeded. The driving force for motion of the contact line is $\gamma_{L V}\left(\cos \theta_{e}-\cos \theta\right)$

\section{${ }_{105}$ Spot size estimates and uncertainties}

A deposited spot will have a contact radius, $r_{o}$, determined by the intial droplet volume, $V_{o}$, and the contact angle, $\theta_{e}$. For small droplets, $r_{o}=(4 / \beta)^{1 / 3} R_{o} \sin \theta_{e}$ where $R_{o}$ is the radius of a hypothetical sphere containing the initial volume of liquid and ${ }_{110} \beta=\left(1-\cos \theta_{e}\right)^{2}\left(2+\cos \theta_{e}\right)$. The upper row in table 1 summarises the effect of a $10 \%$ uncertainty in $R_{o}$ on spot area, $A_{S L}$, for water-on-glass using $\theta_{e}=8^{\circ}$ to $37^{\circ}$. The lower row summarises
Table 1 Spot size variations due to volume and contact angle variations

\begin{tabular}{|c|c|c|c|c|}
\hline$R_{o} / \mu \mathrm{m}$ & $V_{o} / \mathrm{pL}$ & $\theta_{e}{ }^{\circ}$ & $r_{\sigma} / \mu \mathrm{m}$ & $A_{S L} / \mathrm{mm}^{2}$ \\
\hline & & 8 & $101 \pm 10$ & $0.032 \pm 0.007$ \\
$30 \pm 3$ & \multirow{3}{*}{$113 \pm 35$} & 15 & $82 \pm 8$ & $0.021 \pm 0.004$ \\
& & 27 & $67 \pm 7$ & $0.014 \pm 0.003$ \\
& & 37 & $59 \pm 6$ & $0.011 \pm 0.002$ \\
\hline 30 & 113 & 35 & 60.5 & 0.0115 \\
& & 40 & 57.4 & 0.0104 \\
\cline { 3 - 5 } & & & & \\
\cline { 3 - 5 } & & & &
\end{tabular}

the possible variability in the spot area due to uncertainty in the contact angle for water on an aminopropylsilane coated 115 glass slide supplied for microarray usage with a stated value $\theta_{e}=40^{\circ} \pm 5^{\circ}$. In the first case, spot area increases by $50 \%$ from $0.021 \mathrm{~mm}^{2}$ to $0.032 \mathrm{~mm}^{2}$ as the contact angle increases from $8^{\circ}$ to $15^{\circ}$. In the second case, the manufacturer's stated contact angle tolerance of $\pm 5^{\circ}$, translates to $\pm 5 \%$ uncertainty in spot 120 radius and $\pm 10 \%$ in spot area. The general rule is that for $\theta_{e}<60^{\circ}, r_{o} \approx 6.736 R_{o} / \theta_{e}^{1 / 3}$ ( $\theta_{e}$ in degrees) and errors translate as: i) $\%$ error in $r_{o}=\%$ error in $R_{o}$, ii) $\%$ error in $r_{o}=1 / 3^{\text {rd }}$ the $\%$ error in $\theta_{e}$, and iii) $\%$ error in spot area $=$ twice the $\%$ error in $r_{o}$.

125 Substrate surface heterogeneity will result in non-circular spots. If the heterogeneity is chemical, the contact angle is given by the Cassie-Baxter formula: $\cos \theta_{\mathrm{CB}}=f_{1} \cos \theta_{1}+f_{2} \cos \theta_{2}$, where $f_{\mathrm{i}}$ and $\theta_{1}$ are the surface area fraction and contact angle of a patch of the surface of type $\mathrm{i}^{4}{ }^{4}$ For example, an 130 aminopropylsilane coated glass slide $\left(\theta_{1}=40^{\circ}\right)$ with patches $f_{2}=10 \%, 25 \%$ and $50 \%$ of clean hydrophilic glass $\left(\theta_{2}=0^{\circ}\right)$ will give $37.9^{\circ}, 34.5^{\circ}$ and $28.0^{\circ}$, respectively, corresponding to increases in spot areas of $4 \%, 12 \%$ and $31 \%$ compared to that expected without those patches. If there is slight roughness, $135 r_{g}$, then the contact angle is given by Wenzel's formula $\cos \theta_{W}=r_{g} \cos \theta_{1} .{ }^{4}$ For an aminopropylsilane coated glass slide $\left(\theta_{1}=40^{\circ}\right)$ and $r_{g}=1.07(\sim 1 \mathrm{~nm}$ of height in every $30 \mathrm{~nm}$ laterally), the contact angle is decreased to $35^{\circ}$ and the spot area is increased by $\sim 11 \%$. Increasing the roughness to ${ }_{140} r_{g}=1.15$, decreases $\theta_{\mathrm{W}}$ to $28^{\circ}$ and increases spot area by $\sim 30 \%$.

\section{Capillary rise, spotting pins and capillary bridges}

If $\theta_{e}<90^{\circ}$, liquid will rise up the inside of a smooth tube of internal radius, $r$, to a height, $H$, given by $H / \kappa^{-1}=2 \kappa^{-1} \cos \theta_{e} / r$. The strongest effect is for a thin tube. Water will rise $29.9 \mathrm{~cm}$ 145 up a $100 \mu \mathrm{m}$ diameter tube having $\theta_{e}=0^{\circ}$, but only $63 \mu \mathrm{m}$ up the outside of a $20 \mu \mathrm{m}$ diameter fiber of the same material. It is no surprise split, quill and slotted pins are used in microarrays. Using a pin design with a $100 \mu \mathrm{m}$ slot and $20 \mu \mathrm{m}$ exit allows rapid filling and a reservoir to form at the top of 150 the slot so that multiple spots may be deposited without refilling. When a pin contacts a substrate, a rapid downwards and outwards flow occurs, but as the pin is retracted the droplet is pulled up and forms a receding contact angle with the substrate until the capillary bridge between pin and 155 substrate breaks. Controlling the surface energy of the subtrate and pin will control the receding contact angle, the stretching and breakage of the capillary bridge and the final droplet volume and spot size. Spot size will depend on the geometric parameters of the pin and the wetting properties of ${ }_{160}$ the pin and substrate. In the ink-jet approach, capillary bridge 
effects are removed, because it is a non-contact deposition method. However, the instability causing the break-up of the capillary jet and the impact of a droplet on the substrate, still involve surface free energies and so influence spot size.

\section{${ }_{165}$ Excess pressure, spot density and dust}

As spotting density increases, deposited droplets may touch. A droplet on a flat and completely wetting surface $\left(\theta_{e}=0^{\circ}\right)$ spreads into a film because this reduces the Laplace excess pressure, $\Delta P=2 \gamma_{L V} / R$, across its curved liquid-vapor interface 170 of spherical cap radius $R$. When two droplets with different spherical cap radii touch, a capillary bridge forms and the one with the smaller $R$ will flow into the other; droplets with higher curvature have higher execess pressure. In surface free energy terms, coalescing two droplets reduces the total 175 energy. Minimising surface free energy also tells us that dust will always attach to a water-air interface. The more hydrophobic a grain of dust, the more it will project out into the air, but even highly hydrophobic grains attach themselves to a droplet of water. In the extreme case, a droplet of water 180 rolling across a bed of hydrophobic dust will completely selfcoat and become a liquid marble. ${ }^{5}$

\section{Donuts, contact line pinning and mixing}

When droplets are deposited they are three dimensional volumes of liquid, but they dry and so become two 185 dimensional solid spots. The evaporation of a droplet possessing a solute (or coated in particles) will cause a ringstain if the contact line is pinned by contact angle hysteresis; low relative humidity causes fast ring stain formation. ${ }^{6}$ As the droplet evaporates, an internal flow is set-up supplying liquid

190 to the pinned contact line. Hence, suspended solids are carried to the edge of the droplet where most solute deposition then occurs $^{7,8}$ (fig. 3). In microarrays, a ring-stain (or donut) results in non-uniform fluorscence across a spot (fig. 4$)^{6}{ }^{6}$

To suppress ring stains one can try using a high initial 195 solute concentration, keeping the contact angle low (but then spots become large), reducing contact line pinning and ensuring the initial deposition is homogeneous (since there is a strong memory effect of the initial distribution). ${ }^{6}$ Although not a wetting effect, the diffusion time for $1 \mathrm{~mm}$ motion of
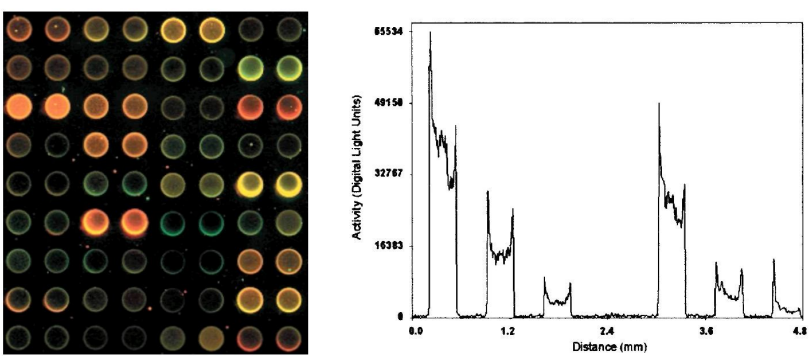

Fig. 4 Non-uniform fluorescence across a spotted microarray due to ring-stain formation (Reprinted with permission from ref. [6]. Copyright 2002 American Chemical Society).

$200100 \mathrm{~nm}$ DNA is thirty hours, which implies a slow hydridisation in a fluid film of $50 \mu \mathrm{m} .{ }^{2}$ Wixforth et al, note that improving uniformity of spot fluorescence is possible using micro-agitation to promote mixing. ${ }^{9}$

\section{Conclusions}

205 Advances in microarray technology now permit combinatorial experiments providing large quantities of data. For data to be of scientific value, we cannot simply process it with sophisticated software and analyze it with careful statistics. We need to understand intrinsic limitations to the accuracy of 210 the data arising from the nature of the interactions. Wetting describes one fundamental interaction, that of the liquid with the solid, and may well influence the quality of our data.

\section{References}

1. L. E. Cheran, M. Chacko, M. Q. Zhang, M. Thompson, Analyst, 2004, 129, 161.

2. A. Wixforth, Superlattices \& MicroStr, 2003, 33, 389

3. D. Stekel, Microarray bioinformatics, Cambridge University Press, 2003 (ISBN 0-521-52587-X)

4. G. McHale, N. J. Shirtcliffe, M. I. Newton, Analyst, 2004, 129, 284.

220 5. P. Aussillous, D. Quéré, D, Nature, 2001, 411, 924.

6. R. Blossey, A. Bosio, Contact Line Deposits on cDNA Microarrays "Twin-Spot Effect" Langmuir, 2002, 18, 2952.

7. R. D. Deegan, Phys. Rev. E, 2000, 61, 475.

8. R. D. Deegan, O. Bakajin, T. F. Dupont, G. Huber, S. R. Nagel, T. A. 225 Witten, Nature, 1997, 389, 827

9. A. Wixforth, C. Strobl, C. Gauer, A. Toegl, Z. v. Guttenberg, Anal. Bioanal. Chem, 2004, 379, 982.
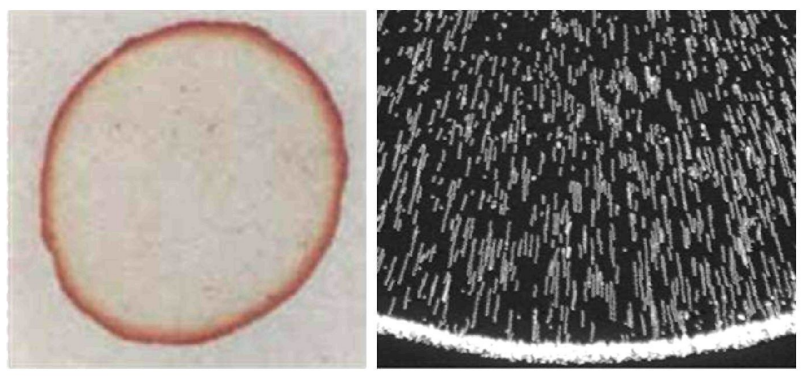

Fig. 3 a) Coffee ring stain (Reprinted Fig. 1a with permission from ref. [7]. Copyright 2006 by the American Physical Society), b) multiple images superimposed to show the flow of solute particles to form a ring stain during evaporation (Reproduced by permission from Macmillan Publishers Ltd [Nature] ref. [8] 1997). 